Project Number: 86804

\title{
Project Title: Resolving the Impact of Biological Processes on DNAPL Transport in Unsaturated Porous Media through Nuclear Magnetic Resonance Relaxation Time Measurements
}

\section{Principal Investigators:}

Dr. Russel Hertzog, Consulting Scientist, Department of Geosciences Research, Idaho National Engineering and Environmental Laboratory, PO Box 1625, Idaho Falls, ID, 83415-2025, Phone number 208-526-4092, Email: hertr@inel.gov

Dr. Gill G. Geesey, Science Fellow, Department of Biotechnology Research, Idaho National Engineering and Environmental Laboratory, PO Box 1625, Idaho Falls, ID, 83415-2025, Phone number 208-526-2233, Email: geesgg@inel.gov

\section{Co-Investigators:}

Dr. Timothy A. White, Staff Scientist, Department of Physics, Idaho National Engineering and Environmental Laboratory, PO Box 1625, Idaho Falls, ID, 83415-2025, Phone number 208-526-8006, Email: whitta@inel.gov

Dr. Clifford K. Ho, Sandia National Laboratory, PO Box 5800, Albuquerque, NM, 87185-0735, Phone number 505-844-2384, Email ckho@sandia.gov
Dr. Christian Straley, Senior Research Scientist, Schlumberger-Doll Research Center, Ridgefield, CT, 06877, Phone 203-431-5412, Email: Straley@slb.com

Dr. Traci R. Bryar, Research Consultant, PO Box 1585, 12 Lloyd-Irwin Street, Marathon, ON P0T2E0, Canada, Phone 807-229-1472, Email: tbryar@hotmail.com

Dr. Joseph Seymour, Assistant Professor, Department of Chemical Engineering, Montana State University, 306 Cobleigh Hall, Bozeman, MT, 59717-3920, Phone number 406-994-6853, Email: jseymour@coe.montana.edu

Dr. Sarah L. Codd, Assistant Research Professor, Department of Chemical and Biological Engineering, Montana State University, 306 Cobleigh Hall, Bozeman, MT, 59717-3920, Phone number 406-994-1944, Email: scodd@coe.montana.edu

Libbie Oram, ACE Fellowship Intern, Idaho National Engineering and Environmental Laboratory, PO Box 1625, Idaho Falls, ID, 83415-2025, Phone number 208-526-1654, Email: oramll@inel.gov

\section{Research Objectives}

This research leads to a better understanding of how physical and biological properties of porous media influence water and dense non-aqueous phase liquid (DNAPL) distribution under saturated and unsaturated conditions. Knowing how environmental properties affect DNAPL solvent flow in the subsurface is essential for developing models of flow and transport that are needed for designing remediation and longterm stewardship strategies. This project investigates the capability and limitations of low-field nuclear magnetic resonance (NMR) relaxation decay-rate measurements for determining environmental properties affecting DNAPL solvent flow in the subsurface. For in-situ subsurface environmental applications, lowfield proton NMR measurements are preferred to the conventional high-field techniques commonly used to obtain chemical shift data, because the low field measurements are much less degraded by the magnetic susceptibility variations between the rock grains and the pore fluids that significantly interfere with the high-field NMR measurements.

Our research scope includes determining whether DNAPLs exist in water-wet or solvent-wet environments, the pore-size distribution of the soils containing DNAPLs, and the impact of biological processes on their transport mechanisms in porous media. Knowledge of the in situ flow properties and pore distributions of organic contaminants are critical to understanding where and when these fluids will enter subsurface aquifers. 


\section{Research Progress and Implications}

As of June 13, this report summarizes work after 9 months of the 3-year project, which consisted of performing initial key experiments, developing rigorous low-field NMR measurement and analysis protocols (decay-time processing techniques), acquiring appropriate and representative native INEEL soils and sands, and developing reproducible column packing and saturating procedures.

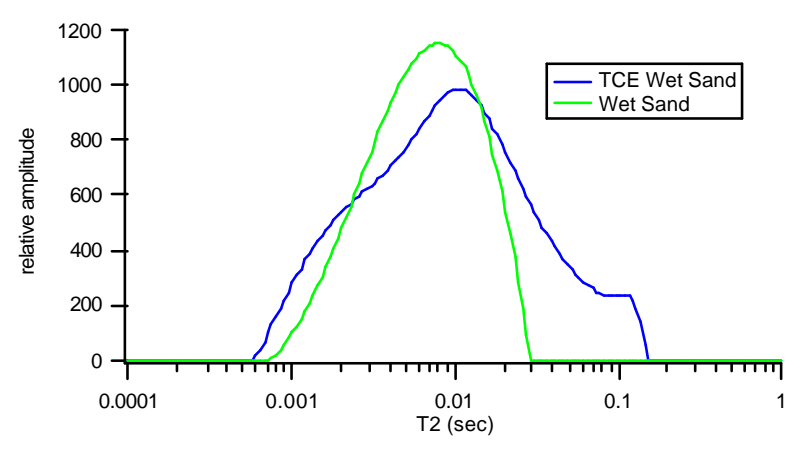
Initial Key Experiments: The first key experiment at INEEL determined that DNAPLs could be detected and distinguished from water in soils. This has been done (completing our first milestone). The $\mathrm{T}_{2}-$ distributions on the left show trichloroethylene (TCE) in a water-wet interbed sand (blue curve) compared to a water-wet interbed sand alone (green curve). Two commonly found subsurface DNAPLs containing hydrogen are TCE and dichloroethylene (DCE), and TCE has the lowest hydrogen index (10\% that of water) and is therefore the most difficult to detect with proton NMR. DCE should provide a signal 2.4 times easier to detect. The second key experiment (Milestone 2) underway uses mixed DNAPL and water (e.g. 50/50 mixed) to establish the saturation and concentration detection limits in porous media.

Other Milestone-2 related key experiments underway concern detecting the effects of bacterial accumulation in saturated and unsaturated porous media on water and DNAPL pore -size distributions. This include the use of synthetic bio-film matrix (poly-saccharic as a surrogate bio-film and sand), the use of biological agents to grow the biofilm, and experiments with multiple (three) pore sizes to determine if biofilm prefers certain pore-size ranges.

The initial stages of the NMR microscopy portion of the project at Montana State University focused on imaging a single biofilm in a $1 \mathrm{~mm}$ capillary reactor. This system serves as a model for a single large anisotropic pore of a porous media and allows for determination of $\mathrm{T}_{2}$, spin-spin magnetic relaxation behavior within the biofilm. The $\mathrm{T}_{2}$ maps shown at right display three slices through a mature Staphylococcus epidermidis biofilm, grown in a $1 \mathrm{~mm}$ square bioreactor for 48 hours [1]. Slice thickness is $300 \mu \mathrm{m}$. Pixel resolution is $20 \mu \mathrm{m} / \mathrm{pixel}$ across the capillary width and $160 \mu \mathrm{m}$ along the length. In the figures the three images are A) bottom slice, B) middle slice, C) top slice, indicating the prevalence of biofilm growth on the bottom of the reactor during the growth period. The imaging method allows for determination of the heterogeneity of $T_{2}$ within the biofilm for use in correlation with future bulk measurements of the same sample that are underway. Model porous media samples composed of $240 \mu \mathrm{m}$

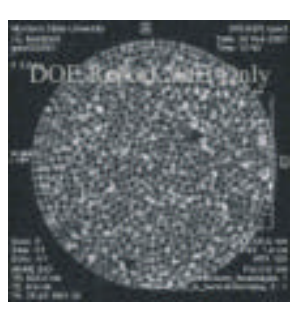
monodisperse polystyrene beads have been constructed and imaging and bulk $\mathrm{T}_{2}$ measurements are underway without biofilm growth to characterize the spatial dependence of $T_{2}$ with the distribution of $T_{2}$ in the bulk measurements of the sample. A $T_{2}$ weighted image of

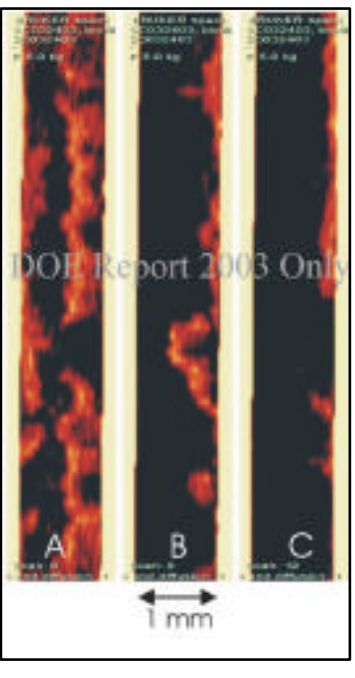
water spin density in the bead pack in a $9 \mathrm{~mm}$ ID sample container is shown at left. The image has pore scale resolution of $40 \mu \mathrm{m} / \mathrm{pixel}$. The methodology for growing biofilm in this sample is currently being developed.

Measurement and Analysis Protocols: The capabilities of the new INEEL low-field MARAN-2 Ultra NMR instrument and the influence of pulse-sequence measurement parameters were characterized and calibrated for various fluid-soil samples to ensure that our results can be duplicated, and are consistent with field tools used in the oil and gas industry. Experimental $\mathrm{T}_{2}$ distributions are very sensitive to the pulse sequence measurement parameters and inversion technique used to analyze the decay-time measurements. A variety 
of calibration liquids and fluid-soil samples were measured and their analyzed results were compared using (1) an inversion package provided by the instrument manufacturer, (2) those used by our collaborators (Whitall, K.P., and Mackay, A.L., "Quantitative interpretation of NMR relaxation data," J. Mag. Res., 84, p. 134-152, 1989), and (3) techniques developed in the oil and gas industry (Straley, C., Rossini, D., Vinegar, H., Tutunjian P., and Morriss, C., "Core analysis by low field NMR," The Log Analyst, Vol. 38, No. 2, p. 84, 1997). The $T_{2}$ distribution shapes are particularly sensitive to the regularization (smoothing) parameter, and a systematic method to control this parameter, based on a quantitative assessment of the quality-of-fit $\chi^{2}$, is being developed to obtain credible $T_{2}$ distributions from any given set of data.

Packing and Saturating Protocols: Two packing protocols were evaluated. The first used extensively by the INEEL hydrology research community consists of partially filling a soil column with sand, saturating with water, and compacting the sand by setting the column on a vacuum plate and removing the excess water. This process is repeated until the column is full. A centrifuge is used to pack to the desired porosity. The column is dried, and then saturated with water or DNAPL with a vacuum system. An alternate packing and saturating procedure developed by the oil and gas industry was chosen for our initial packing protocol. It consists of filling columns with enough fluid to fill the pore space, adding sand to partially fill the column, and vibrating the column to pack the sand. This procedure is repeated until the column is full. Pre-filling with fluid ensures that there are no gas bubbles in the pore space. Both procedures use mass-balance to determine fluid volume, sand volume, and porosity, and can be modified to build water-wet or DNAPL-wet saturated columns. These methods work well for manufactured sand samples (silicon-oxide doped with iron). However, the vibrating table tends to sort sands with a wide range of grain sizes into layers and may not be appropriate for INEEL-site sands.

Paramagnetic-ion Doping Procedure for Silica (pure) Sand: The ultra-pure (99.995\%) silica sand will be cleaned by soaking it in $10 \% \mathrm{HCl}$ and rinsing it thoroughly with de-ionized water. The relaxation time of the water in the saturated sand will be tested and the process repeated until $\mathrm{T}_{2}$ becomes constant, ensuring ferromagnetic impurities have been removed from the sand. To coat the sand, a modification of the method in Grantham, M.C., Dove P.M., and DiChristina, T.J., (Geochimica et Cosmochimica Acta, Vol 61, 4467 $4477,1997)$ will be used. The clean sand will be mixed with a solution of $\mathrm{FeSO}_{4}(0.001-0.1 \mathrm{M}$, depending on the desired surface concentration) and after $30 \mathrm{~min}, 30 \% \mathrm{H}_{2} \mathrm{O}_{2}$ will be added to oxidize the $\mathrm{Fe}$ (II) to $\mathrm{Fe}(\mathrm{III})$. The solution will be mixed well and allowed to stand overnight enabling a FeOOH precipitate to form after which the sand will be filtered and dried. If more Fe(II) than available surface sites is added, excess $\mathrm{FeOOH}$ will form a fine precipitate that can easily be separated from the sand during filtering. The sand will be analyzed for $\mathrm{Fe}$ and surface area along with site sediments utilizing in-house facilities.

\section{Planned Activities}

The anticipated outcome of this research will establish the utility of proton NMR measurements for: a) elucidating DCE and TCE pore size distribution and flow properties in different porous media, b) detecting a microbiological influence on DNAPLs, and c) DNAPL pore size distribution under saturated and unsaturated conditions. Current planned activities include the following with associated schedules:

- Complete DNAPL detection and characterization limits

- Detect effects of bacteria accumulation in porous media on water/DNAPLs

December 2003

- Detect influence of biofilm in unsaturated media

Ongoing

- Determine size distributions of INEEL soils near DNAPL plumes December 2003

- Distinguish water-wet and DNAPL-wet soils 2004

- Assess microorganism influence on DNAPLs in saturated and unsaturated media 2003-2004

- Meso-scale experiments to verify scalability of column studies 2004-2005

- Field tests with commercial NMR logging tools

2005

\section{Information Access:}

Seymour, J.D., Codd, S.L., Gjersing, E.L., Busse, S.C., and Stewart, P.S., "NMR microscopy of the impact of biofilm structure on transport in a capillary bioreactor," J. Mag. Res. to be submitted (2003). 